\title{
A Biased Agonist at Immunometabolic Receptor GPR84 Causes Distinct Functional Effects in Macrophages
}

\author{
Daniel Lucy, ${ }^{\dagger \neq}$ Gareth S. D. Purvis, ${ }^{\ddagger}$ Lynda Zeboudj, ${ }^{\ddagger}$ Maria Chatzopoulou, ${ }^{\dagger}$ Carlota Recio, \\ Carole J. R. Bataille,,$^{\dagger}$ Graham M. Wynne, ${ }^{\dagger}$ David R. Greaves, ${ }^{*},+$ and Angela J. Russell $*^{\dagger},, \S_{(0)}$ \\ ${ }^{\dagger}$ Department of Chemistry, University of Oxford, Mansfield Road Oxford OX1 3TA, U.K. \\ ${ }^{\ddagger}$ Sir William Dunn School of Pathology, University of Oxford, South Parks Road, Oxford OX1 3RE, U.K. \\ ${ }^{\S}$ Department of Pharmacology, University of Oxford, Mansfield Road, Oxford OX1 3QT, U.K.
}

\section{Supporting Information}

ABSTRACT: GPR84 is an orphan G-protein-coupled receptor that is expressed on immune cells and implicated in several inflammatory diseases. The validation of GPR84 as a therapeutic target is hindered by the narrow range of available chemical tools and consequent poor understanding of GPR 84 pathophysiology. Here we describe the discovery and characterization of DL-175, a potent, selective, and structurally novel GPR84 agonist and the first to display significantly biased signaling across GPR84-overexpressing cells, primary murine macrophages, and human U937 cells. By comparing DL-175 with reported GPR84 ligands, we show for the first time that biased GPR84 agonists have markedly different abilities to induce chemotaxis in human myeloid cells, while causing similar levels of phagocytosis enhancement. This work demonstrates that biased agonism at GPR84 enables the selective activation of functional responses in immune cells and delivers a high-quality chemical probe for further investigation.

-protein-coupled receptors (GPCRs) expressed on the $J$ surface of immune cells control cellular functions that are critical for the maintenance of immune homeostasis and the mounting of inflammatory responses. While some immune GPCRs, such as the chemokine receptors, have been the subject of extensive research and actively pursued as drug targets, other GPCRs expressed throughout the immune system remain poorly characterized and represent a relatively unexploited area for treatments of inflammatory diseases. ${ }^{1}$ The free fatty acid receptors (FFARs) are a subset of four immuneexpressed GPCRs with metabolic intermediary fatty acids as their endogenous ligands, suggesting a role for dietary fatty acids in the regulation of immunity and inflammation. ${ }^{2,3}$ The clinical potential of these receptors has been demonstrated by the small molecule agonist of the free fatty acid receptor 1 , fasiglifam, which showed efficacy in a Phase II trial for diabetes.

GPR84 is the putative fifth fatty acid receptor and is highly expressed in the cells of myeloid lineage that constitute the innate immune system, including monocytes, macrophages, and neutrophils in the periphery and microglia in the brain., Saturated fatty acids with chain lengths between C9 and C14 are activators of the receptor, although the weak potency of this interaction means that GPR84 officially remains an orphan receptor. ${ }^{7}$ GPR84 expression in leukocytes is markedly upregulated by acute inflammatory stimuli, such as lipopolysaccharide (LPS), and tissue from mice exposed to hyperglycemic or dyslipidemic conditions associated with chronic low-grade inflammation also shows elevated GPR84 mRNA. ${ }^{8,9}$ Activation of the receptor in macrophages has been shown to result in increased cytokine secretion, immune cell migration, and enhanced phagocytosis. ${ }^{7,9,10}$ These studies of GPR84 biology almost exclusively use a narrow range of fatty acids or fatty acid mimetics to activate the receptor, which may not reflect the physiological activation of GPR84 in vivo, given the low potency of the purported natural fatty acid agonists. The possibility of other, as yet undiscovered, endogenous GPR84 ligands increases concern that additional aspects of GPR84 function may be hidden without the use of more diverse chemical tools.

A number of surrogate agonists for GPR84 have been identified in addition to fatty acids (Figure 1), including natural product embelin (2), ${ }^{11}$ synthetic compound 6octylaminouracil (6-OAU, 3), ${ }^{10}$ and structurally related compounds with improved potency, 4 and 5. ${ }^{12,13}$ All reported orthosteric GPR84 agonists conform to a fatty acid mimetic structure with polar head groups appended to long alkyl or lipophilic chains, although 3,3-diindolylmethane and derivatives have been reported as ago-allosteric GPR84 modulators, ${ }^{14}$ and dihydropyrimidinoisoquinolinones are described in the patent literature as GPR84 antagonists. ${ }^{15}$ Recently, a

Received: July 4, 2019

Accepted: August 29, 2019

Published: August 29, 2019 


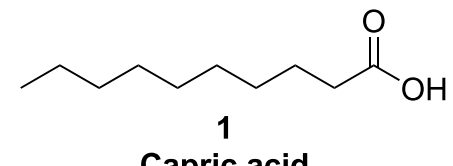

Capric acid<smiles>CCCCCCCCNc1cc(=O)[nH]c(=O)[nH]1</smiles><smiles>CCCCCCCCCc1cc(O)cc(=O)[nH]1</smiles><smiles>CCCCCCCCCCCC1=C(O)C(=O)C=C(O)C1=O</smiles><smiles>CCCCCCSc1nc(O)cc(=O)[nH]1</smiles><smiles>O=c1cc(NCCc2ccc(Cl)cc2)[nH]c(=O)[nH]1</smiles>

Figure 1. Chemical structures of selected GPR84 agonists. Compounds 1-4 are commonly used GPR84 tool agonists. Compound 5 has been reported to have sub-nanomolar activity at the receptor, and $\mathbf{6}$ is reported to have G-protein signaling bias.

series of uracil derivatives with potent activity at GPR84 were reported, including some compounds, such as 6, with significant G-protein signaling bias. ${ }^{16}$ Biased agonism is an emerging field in the study of GPCRs in which agonists for the same receptor can bring about different patterns of downstream signaling. In particular, examples of agonists that selectively activate canonical G-protein pathways or noncanonical $\beta$-arrestin dependent pathways have been reported, enabling the separation and selective induction of functional effects where they correspond to a specific pathway. ${ }^{17}$ The limited range of GPR84 agonists and paucity of examples with significant signaling bias has meant that the implications of biased agonism at GPR84 on immune cell behavior or function have not yet been explored.

In this study, we set out to expand the range of tools available for investigating GPR84 biology and used a virtual screening strategy to discover a structurally novel agonist, DL175 , that exhibits biased signaling in GPR84-CHO cells. We show that this signaling bias translates into primary murine macrophages and human U937 macrophages, where DL-175 causes unique responses in impedance signaling assays. Finally, we show that DL-175 and literature GPR84 agonist 6-OAU both cause similar phagocytosis enhancement in U937 macrophages but have drastically different abilities to induce directed migration in U937 macrophages and primary human monocytes. The functional consequences of GPR84 activation are therefore dependent on the ligand used, with significant implications for attempts at using small molecule tools to probe the pathophysiological role of GPR84.

\section{RESULTS AND DISCUSSION}

Discovery of a Novel GPR84 Agonist. In the absence of a published crystal structure of GPR84, we used a ligand-based virtual screening methodology to identify novel GPR84 agonists (Figure 2a). A literature data set comprising 32 compounds structurally similar to 6-OAU was used to construct a predictive quantitative structure-activity relationship (QSAR) model (Forge, Cresset). ${ }^{13}$ Then, 10,000 structurally diverse compounds from an in-house library were screened against the model, and 45 compounds selected on the basis of predicted potency and fit to the model were tested in a GPR84-CHO cAMP inhibition assay (Supplementary Table 1). Several hit compounds were identified, including fatty acid mimetics with nanomolar potency. GPR84 active compounds were counter-screened against $\mathrm{CHO}$ cells expressing the $\mathrm{G}_{i}$ coupled $\mathrm{CB}_{1}$ or $\mathrm{G}_{\mathrm{s}}$ coupled $\beta_{2}$-adrenergic GPCRs, in addition to untransfected $\mathrm{CHO}$ cells (Supplementary Table 2). Among the hit compounds with genuine activity at GPR84, chloronaphthol imidazole 7 was notable as a novel scaffold of GPR84 agonist (Supplementary Figure $1 \mathrm{a}-\mathrm{c}$ ) and was therefore chosen for further investigation.

We next set out to optimize the small molecule hit 7 into a compound with suitable properties for use as a chemical probe for GPR $84{ }^{18}$ Initially, a preliminary medicinal chemistry optimization study (Figure $2 b$ ) was performed to enhance the potency of the compound series. Replacing the imidazole heterocycle with pyrazole $(8)$ or pyridyl regioisomers (9 and 11) resulted in a complete loss of activity. 3-Pyridyl 10, however, showed enhanced activity and suggested the importance of a hydrogen bond acceptor in the 3-position of the heterocyclic moiety. Oxidation of 4-pyridyl 9 to the equivalent $\mathrm{N}$-oxide 12 improved potency, while oxidation of 3pyridyl 10 led to the most potent compound in the series, DL175 (13), which has activity akin to that of 6-OAU. The GPR84 specificity of the observed inhibition of cAMP accumulation by DL-175 in GPR84-CHO cells was confirmed by the absence of response in untransfected $\mathrm{CHO}$ cells (Supplementary Figure 1d). Regioisomer 2-pyridine $\mathrm{N}$-oxide 14 fails to activate GPR84 and was also confirmed not to inhibit the receptor (data not shown). Compound 14 can therefore serve as a useful, structurally related, inactive control to DL-175.

To establish the GPR84 selectivity of DL-175 against a broad range of receptors, we screened the compound in GPCR profiling panels. In a panel of 14 human GPCRs covering multiple receptor families, DL-175 $(3 \mu \mathrm{M})$ showed no significant activation of any receptors in cAMP or $\mathrm{Ca}^{2+}$ flux functional assays (Supplementary Figure 2a). Notably, no activity was observed at the free fatty acid receptors (FFAR 14) which might be expected to have similar ligand binding sites to GPR84 given their similar lipid agonists. In a different 


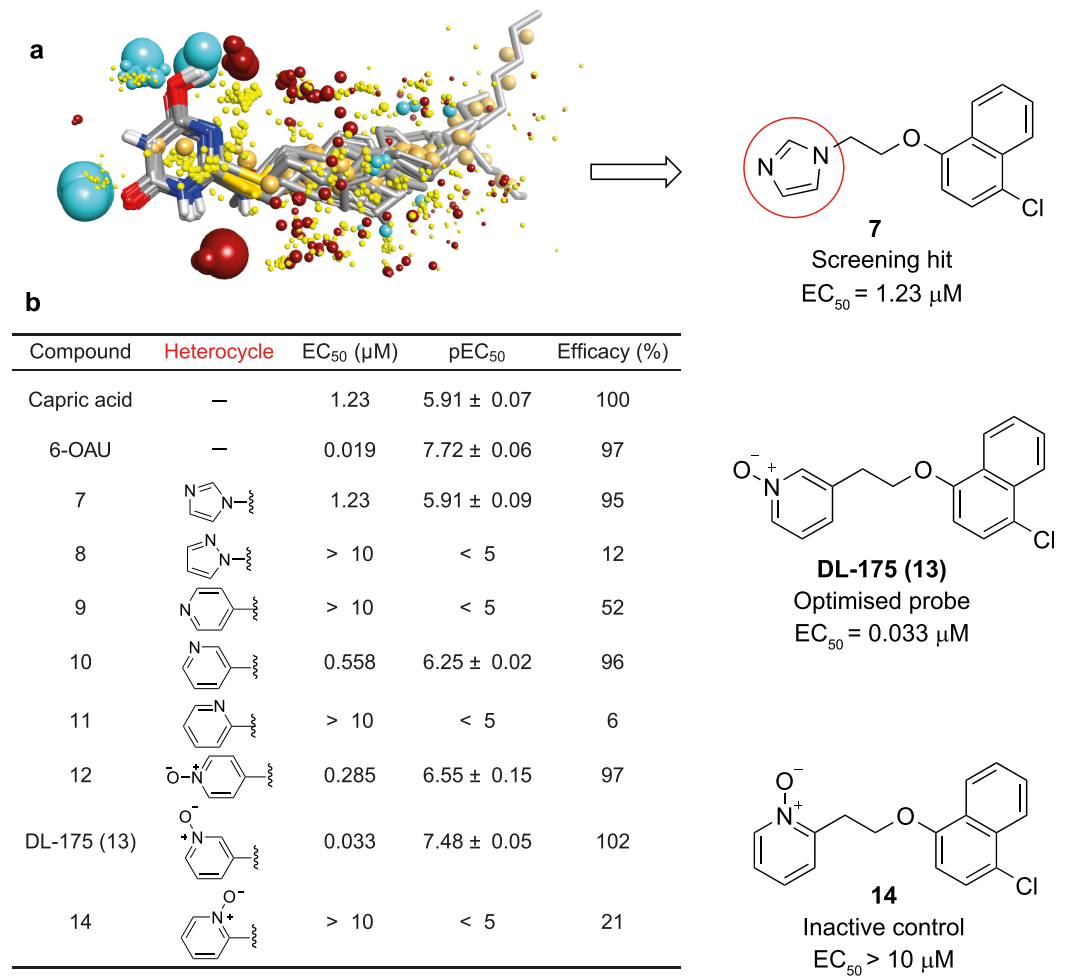

Figure 2. Discovery and optimization of a novel GPR84 agonist. (a) Overlay of 32 compounds used to generate 3D-QSAR model with common areas of positive charge (red), negative charge (blue), and hydrophobic regions (gold) highlighted. The structure of the initial screening hit 7 is shown with the indicated heterocyclic area of focus for the preliminary structure-activity relationship study. (b) Activity of compounds with imidazole heterocycle replacements in GPR84-CHO cAMP assay. Efficacy is relative to the maximum effect of capric acid (100 $\mu \mathrm{M})$. Data shown as means \pm SEM of $n=3-4$ independent experiments.

GPCR panel using $\beta$-arrestin recruitment assays, DL-175 (3 $\mu \mathrm{M}$ ) was inactive at all 168 GPCRs tested (Supplementary Figure $2 \mathrm{~b}$ ). Moreover, in antagonist mode, pretreatment with 3 $\mu \mathrm{M}$ DL-175 did not significantly inhibit the activation of any of the 168 GPCRs with their respective reference agonists (Supplementary Figure 2c). DL-175 therefore has a clean selectivity profile across a wide range of GPCR targets and in both second messenger functional assays and $\beta$-arrestin recruitment assays.

We further characterized DL-175 and its inactive counterpart 14 using chemical and metabolic stability studies. Incubating DL-175 and 14 at $37{ }^{\circ} \mathrm{C}$ in neutral ( $\left.\mathrm{pH} 7.4\right)$, acidic ( $\mathrm{pH} 2.5$ ), or basic ( $\mathrm{pH} 10)$ buffers resulted in negligible degradation after $3 \mathrm{~h}$ (Supplementary Figure 3a,b). In the case of DL-175, more than $80 \%$ of compound remained even after $48 \mathrm{~h}$, and regardless of $\mathrm{pH}$. In metabolic clearance assays (Supplementary Figure 3c,d), DL-175 showed good stability in murine $S 9$ liver microsomes $\left(t_{1 / 2}=73 \mathrm{~min}\right)$ but was rapidly cleared when exposed to whole mouse hepatocytes $\left(t_{1 / 2}<10\right.$ min), likely precluding its use as an in vivo tool compound. The high potency, selectivity, and chemical stability of DL-175, coupled with its inactive negative control 14, make it an ideal chemical probe for studying GPR84 biology in vitro.

DL-175 Fails to Recruit $\beta$-Arrestin in GPR84-CHO Cells. To understand if the structural novelty of DL-175 confers unique pharmacology at GPR84, we compared it to the most commonly used chemical tool agonist, 6-OAU, in GPR84-CHO cell signaling assays. In inhibition of cAMP accumulation assays, the two ligands show comparable potency and efficacy (Figure 3a). To investigate the capacity of the ligands to activate alternative downstream signaling pathways, we monitored the recruitment of $\beta$-arrestin 2 following receptor activation using $\beta$-galactosidase enzyme fragment complementation technology (PathHunter, DiscoverX). ${ }^{19}$ The reduced signal amplification of this assay format required relatively high concentrations of agonist to achieve saturating responses, so a nontoxic detergent was used to prevent aggregation of DL-175 and 14 causing spurious positive results (Supplementary Figure 4). ${ }^{20}$ 6-OAU stimulation induced a luminescent signal corresponding to GPR84 and $\beta$-arrestin association in a dose-dependent fashion (Figure 3b). Similarly, other GPR84 agonists embelin, ZQ-16, and 5 gave responses in the expected rank order of potency (Supplementary Figure 5). ${ }^{16}$ In contrast, DL-175 produces no response even at the highest concentration tested, suggesting a bias for G-protein signaling pathways (Figure $3 \mathrm{~b}$ ). We therefore compared the activity of DL-175 to a previously reported $\mathrm{G}_{\mathrm{i}}$ biased GPR84 agonist, PSB-16434 (6). DL-175 showed much less activation of arrestin signaling than PSB-16434 despite their relatively small difference in cAMP potency, consistent with DL-175 possessing significant signaling bias (Supplementary Figure $6 a, b)$.

To further validate the G-protein bias of DL-175, we examined if the compound would block the action of a full $\beta$ arrestin agonist in GPR84-CHO cells. Preincubation with DL175 reduced $\beta$-arrestin recruitment to GPR84 in response to 6OAU (Supplementary Figure 6c), consistent with effective antagonism of arrestin signaling by DL-175. Finally, as efficacious $\beta$-arrestin recruitment can require the expression of G-protein-coupled receptor kinase 2 (GRK2), we investigated if hGRK2 transfection would reveal DL-175 agonism of $\beta$-arrestin pathways. In fact, DL-175 remained 
a

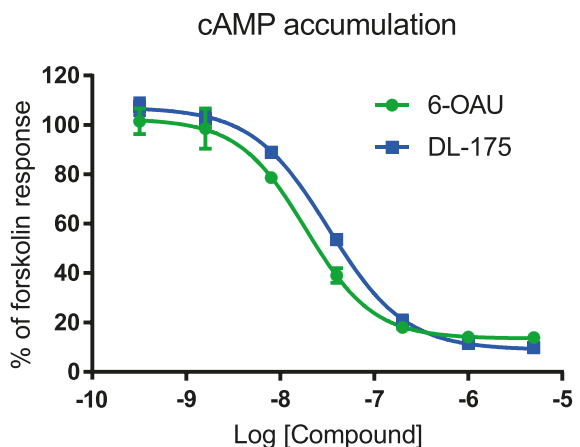

b

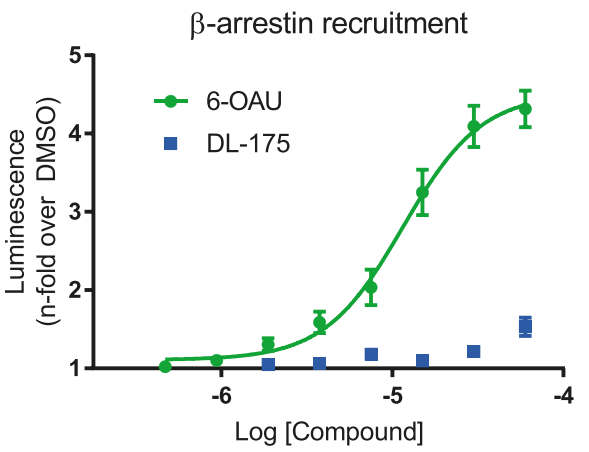

Figure 3. DL-175 is a G-protein biased agonist in GPR84 transfected CHO cells. (a) Dose-response of 6-OAU and DL-175 in an assay measuring the inhibition of forskolin induced cAMP accumulation in GPR84-CHO cells. 6-OAU EC $50=19 \mathrm{nM}$; DL-175 EC $50=33 \mathrm{nM}$. Data pooled from $n$ $=3$ independent experiments and plotted as means \pm SEM of the percentage of the response to forskolin in the absence of agonists. (b) Doseresponse of agonists in a GPR84-CHO $\beta$-arrestin recruitment assay in the presence of $0.025 \%$ Tween-80. 6-OAU EC $50=11 \mu \mathrm{M}$; DL-175 EC 50 $60 \mu \mathrm{M}$. Data plotted as the $n$-fold increase in luminescence over the response to DMSO and shown as means \pm SEM from $n=3$ independent experiments.

entirely inactive for $\beta$-arrestin translocation in GPR84-CHO cells that overexpressed hGRK2 (Supplementary Figure 7). 6OAU retained activity but surprisingly showed reduced efficacy in cells with enhanced GRK2 expression, suggesting that other GRK isoforms such as GRK3, GRK5, and GRK6 are primarily involved in phosphorylation of GPR84 and subsequent arrestin recruitment.

The absence of detectable $\beta$-arrestin recruitment induced by DL-175 prevents the calculation of formal bias factors, but there is a clear preference for G-protein signaling pathways in comparison to 6-OAU. Given the significant difference in signaling pathway activation between the two ligands, a direct comparison will enable investigation of the consequences of functional selectivity at GPR84 and help in understanding the role of $\beta$-arrestin recruitment in creating functional responses following receptor stimulation.

DL-175 Induces Novel Signaling in Macrophages. The downstream signaling induced by GPR84 agonists in transfected cell systems has been shown to poorly reflect the signaling observed in primary immune cells. ${ }^{11}$ For this reason, we chose to further investigate our biased GPR84 agonist in primary murine bone marrow-derived macrophages (BMDMs) that express the receptor and associated signaling machinery at physiological levels. A label-free electrical cell impedance assay that enables rapid morphological changes to be monitored in real-time was employed to give an integrated readout of cellular signaling following GPR84 stimulation. ${ }^{21}$

As the expression of GPR84 mRNA in macrophages is heavily regulated by their activation state, ${ }^{8,9}$ initially GPR84 agonists were tested in differentially polarized BMDMs, and cellular impedance was recorded (Figure 4a,b). Both 6-OAU and DL-175 induced a rapid response in the BMDMs at $1 \mu \mathrm{M}$, with the Cell Index (CI) measure of cellular impedance peaking within $10 \mathrm{~min}$ before decaying to baseline at different rates. For both compounds, the greatest response was observed in LPS stimulated, pro-inflammatory M1 macrophages, with smaller responses observed in alternatively activated IL-4 stimulated M2 cells, and unstimulated M0 cells. These results correlate well with previously reported GPR84 mRNA expression data, and subsequent experiments were performed with LPS stimulated macrophages to achieve maximal responses. 9
Quantifying the M1 polarized BMDM impedance traces by the magnitude of the initial response demonstrated that 6OAU $\left(\mathrm{EC}_{50}=129 \mathrm{nM}\right)$ and DL-175 $\left(\mathrm{EC}_{50}=50 \mathrm{nM}\right)$ have comparable potencies in primary macrophages (Figure $4 \mathrm{c}$ ), as expected from the similar $\mathrm{EC}_{50}$ values observed in transfected cell cAMP accumulation assays. The GPR84 specificity of these responses at $1 \mu \mathrm{M}$ was demonstrated by their absence in GPR84-knockout macrophages, which respond normally to complement component C5a (Figure 4d,e). We next compared the impedance trace of DL-175 to a range of published GPR84 ligands. Each of the structurally similar agonists 6-OAU, ZQ-16, and 5 (Figure 1) induces a rapid increase in cell index, which returns to the baseline within 10 min (Figure 4f). In contrast, DL-175 causes a sustained response in which the cell index remains elevated for over 30 min. Plotting the area under the curve of the impedance traces illustrates the differences between agonists and suggests that novel signaling is occurring with DL-175 (Figure $4 \mathrm{~g}$ ). The inactive DL-175 analogue 14 fails to induce any response in the BMDMs as expected. As GPCR mediated impedance responses are linked to changes in the actin cytoskeleton, ${ }^{22}$ it is likely that DL-175 causes alternative cytoskeletal rearrangement when compared to other GPR84 agonists.

We next set out to establish if the difference in responses observed for the G-protein biased agonist DL-175 were a consequence of the absence of $\beta$-arrestin signaling. First, we used the $\mathrm{G}_{\alpha \mathrm{i}}$ inhibitor pertussis toxin (PTX) to block the expected G-protein signaling pathway. The responses to both 6-OAU and DL-175 were ablated by PTX pretreatment (Figure $4 \mathrm{~h}, \mathrm{i}$ ), suggesting that $\mathrm{G}_{\alpha \mathrm{i}}$ protein engagement is essential for creating the cytoskeletal response. This is consistent with reports suggesting that $\beta$-arrestin signaling alone is not detectable with label-free biosensor assays. ${ }^{23}$ To investigate if $\beta$-arrestin interactions could modify the shape of the impedance response, we employed an inhibitor of Gprotein-coupled receptor kinases (GRK) 2 and 3, CMPD101, that would be expected to limit phosphorylation of GPR84 and thus indirectly prevent the recruitment of $\beta$-arrestin to the receptor. $^{24}$ We treated BMDMs with CMPD101 at various concentrations and then measured the impedance response to 6-OAU or DL-175 stimulation (Supplementary Figure 8a,b). Quantification by measuring the AUC demonstrates that increased GRK inhibition is associated with more sustained 


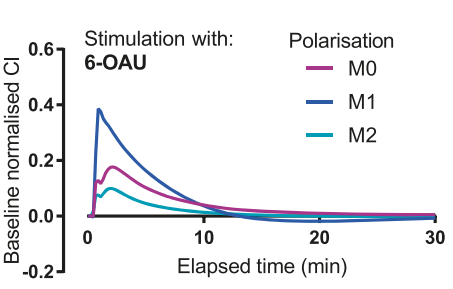

d

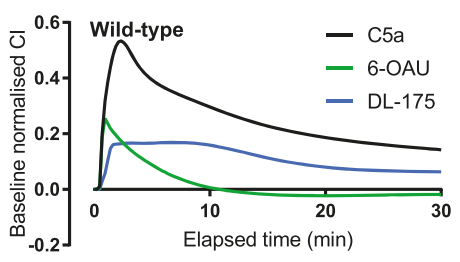

9

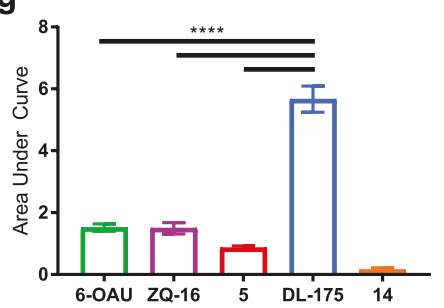

b

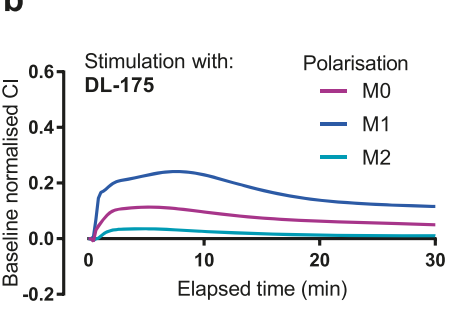

e

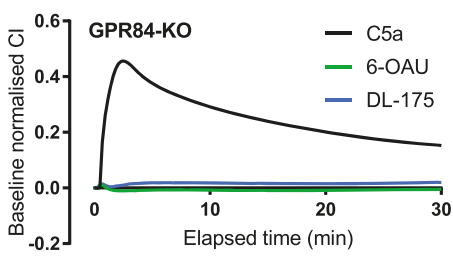

h

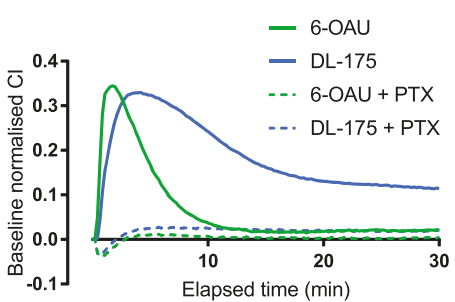

c

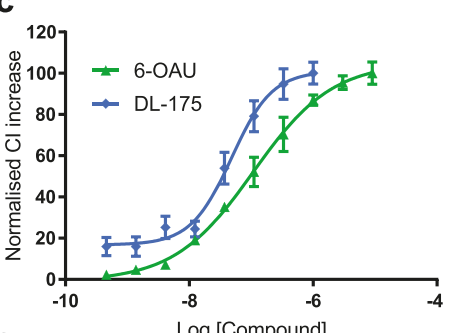

$\mathbf{f}$

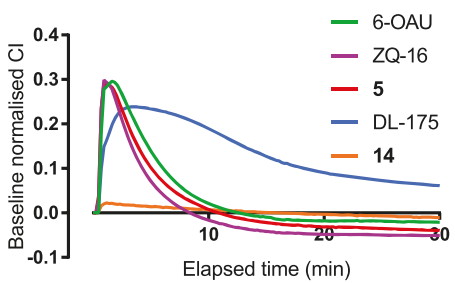

i

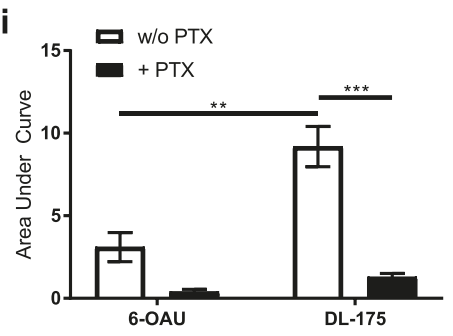

Figure 4. DL-175 induces different responses in primary macrophages compared to other GPR84 agonists. (a) Impedance traces of differentially polarized BMDMs ( $\mathrm{M} 1=16 \mathrm{~h}$ LPS pretreatment; M2 $=16 \mathrm{~h} \mathrm{IL}-4$ pretreatment; M0 = vehicle pretreatment) following stimulation with $1 \mu \mathrm{M}$ GPR84 agonists (a) 6-OAU and (b) DL-175. (c) Normalized dose-response curves generated by quantifying impedance traces of M1 polarized BMDMs according to the magnitude of the initial peak. 6-OAU EC $\mathrm{E}_{50}=129 \mathrm{nM}$; DL-175 EC $50=50.3 \mathrm{nM}$. (d) Wild-type M1 polarized BMDMs respond to C5a $(10 \mathrm{nM})$ and GPR84 agonists $(1 \mu \mathrm{M})$, and (e) the response to GPR84 agonists is ablated in GPR84-KO macrophages. (f) BMDM impedance traces for several GPR84 agonists $(1 \mu \mathrm{M})$ and inactive control 14. (g) Quantification of data in panel $\mathrm{f}$ showing area under the curve for 60 min following agonist addition. (h) Pretreatment with PTX $(200 \mathrm{ng} / \mathrm{mL})$ for $16 \mathrm{~h}$ blocks impedance response of M1 polarized BMDMs to 6OAU and DL-175 $(1 \mu \mathrm{M})$ as illustrated in the quantification in panel i showing area under the curve up to 60 min. Impedance traces are shown as the pooled means from $n=3-4$ biological replicates, and dose-responses and quantifications are shown as pooled means \pm SEM from 3-4 biological replicates. Statistical analysis was conducted using one-way ANOVA with Sidak's multiple comparison test. ${ }^{*} P<0.05$, $* * P<0.01, * * * P$ $<0.001, * * * * P<0.0001$ for indicated comparisons.

responses to 6-OAU, such that at high concentrations of CMPD101, the 6-OAU response resembles that of DL-175. GRK2/3 inhibition also results in a more sustained response to DL-175, although the effect is much less pronounced than for 6-OAU (Supplementary Figure 8c,d). These data suggest that differences in GRK regulation of GPR84 signaling underlie the singular macrophage response to DL-175. Both $\beta$-arrestin 2 and GRK2 are expressed in pro-inflammatory macrophages; ${ }^{25}$ however arrestin knockdown experiments will be required to confirm if GPR84-arrestin interactions induced by GRKs are directly responsible for our cell impedance observations.

To determine if human macrophage populations would also exhibit differing responses to the GPR84 agonists, we used PMA differentiated human U937 macrophage-like cells that express GPR84 to a similar level as primary macrophages. ${ }^{26}$ As expected, DL-175 stimulation of M1 polarized U937 cells resulted in a significantly more sustained impedance response than treatment with 6-OAU (Supplementary Figure 9). Inactive analogue 14 elicited no response, while preincubation with a reported GPR84 antagonist ${ }^{9,15}$ blocked all responses except that to C5a, confirming the specificity of the induced signal. The differential signaling observed with DL-175 therefore extends to a human myeloid cell line.
DL-175 and 6-OAU Cause Different Functional Responses in Immune Cells. As cytoskeletal rearrangement is fundamental to the initiation of macrophage functional responses such as phagocytosis and chemotaxis, ${ }^{27,28}$ we next investigated if biased GPR84 agonists might show differences in their regulation of these processes. We have previously shown that 6-OAU activation of GPR84 in murine macrophages is associated with enhanced phagocytic capacity. ${ }^{9}$ To see if DL-175 would have similar effects on phagocytosis, we used flow cytometry to measure the uptake of opsonized FITC labeled $2 \mu \mathrm{m}$ beads in M1 polarized U937 macrophages (Figure $5 \mathrm{a}-\mathrm{d}$ ). Stimulation with both 6-OAU and DL-175 (1 $\mu \mathrm{M})$ prior to addition of beads led to a small but statistically significant augmentation of bead phagocytosis (Figure 5e,f), which GPR84 inactive DL-175 analogue 14 failed to replicate. To confirm that we were measuring phagocytosis of the beads and not simply adhesion, we used a confocal fluorescence microscopy Z-stack to show that the beads were fully encapsulated within the cell (Supplementary Figure 10). Both GPR84 agonists therefore show similar effects on human U937 macrophage phagocytosis of opsonized beads, despite their different signaling bias.

We next determined the ability of the GPR84 agonists to act as chemoattractants, given the literature describing GPR84 as a 


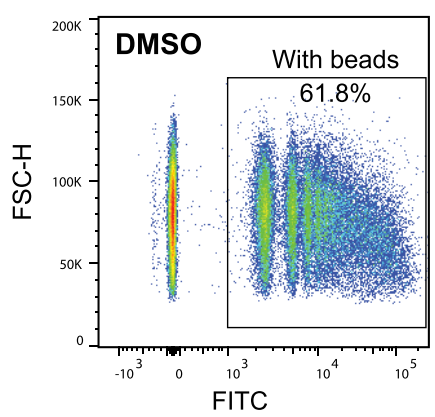

C

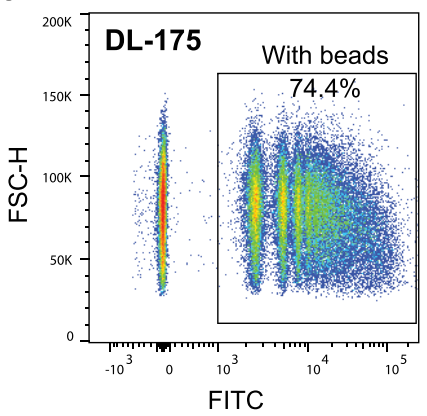

b

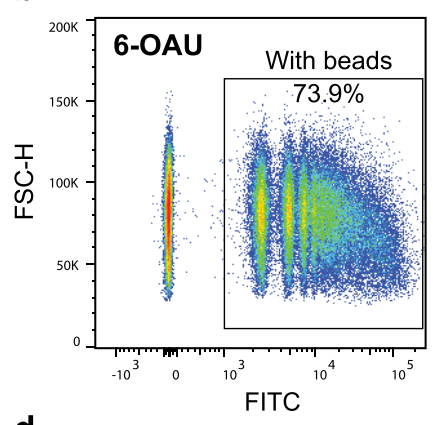

d

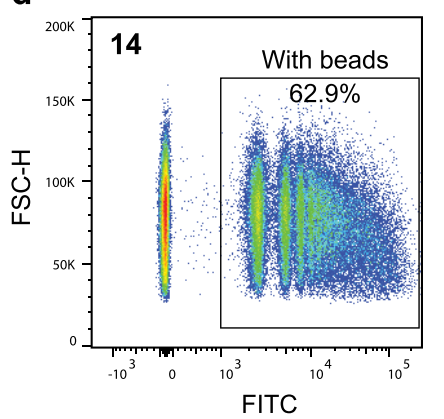

e

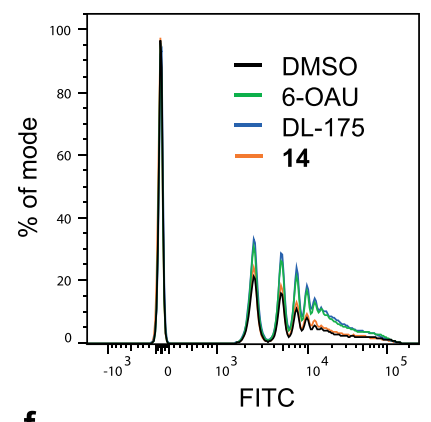

f

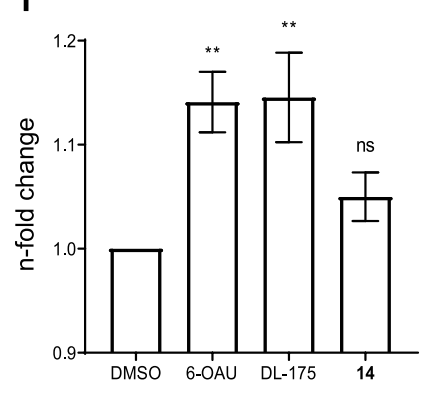

Figure 5. 6-OAU and DL-175 both enhance phagocytosis in U937 macrophages. (a-d) Representative flow cytometry plots of U937 cells incubated with fluorescently labeled beads and different compound pretreatments. Cells with beads are gated according to FITC fluorescence. (e) Representative histogram showing the frequency of cells with different levels of fluorescence. Peaks corresponding to different numbers of phagocytosed beads are visible. (f) Pooled data showing the percentage of cells with beads as $n$-fold change over DMSO. Data are means \pm SEM for $n=6$ independent experiments. Statistical analysis was conducted by one-way ANOVA with Sidak's multiple comparisons correction. ns $=$ not significant, $* * P<0.01$ compared to DMSO treatment.

chemotactic receptor in various immune cells. ${ }^{10,15}$ For this, we used a modified Boyden chamber assay where cells seeded into an upper chamber migrate along a chemoattractant gradient through a porous membrane, before adhering to an electrode in the lower chamber. ${ }^{29}$ The migration of cells can therefore be observed in real time by increases in the cell index measure of cellular impedance.

6-OAU provoked chemotaxis of M1 polarized U937 macrophages with a classical bell-shaped concentration dependence that is characteristic of chemotaxis (Figure $6 a, b) .{ }^{30}$ In contrast, G-protein biased agonist DL-175 failed to induce significant migration of U937 cells at the same concentrations despite their comparable potencies in GPR84CHO cAMP assays. The GPR84 specificity of the 6-OAU induced migration was demonstrated by pretreatment with a GPR84 antagonist, which blocked migration of U937 cells to 6-OAU, while not affecting migration to complement component C5a (Figure 6c,d). Pretreatment with $G_{i / o}$ inhibitor PTX also blocked U937 cell chemotaxis toward 6$\mathrm{OAU}$, indicating that G-protein signaling is required for GPR84 mediated migration. The failure of G-protein biased DL-175 to promote macrophage chemotaxis suggests that additional signaling machinery beyond G-proteins must also be engaged following GPR84 stimulation to enable a migratory response. Biased signaling at the receptor therefore enables the selective activation of functional responses in human U937 macrophages.

Finally, we investigated the responses of primary human monocytes to the two GPR84 agonists. Monocytes express GPR84 in their basal state, but the role of the receptor in promoting monocyte chemotaxis has not yet been described despite the importance of the process in chronic disease states such as atherosclerosis. ${ }^{31} \mathrm{We}$ used magnetic-activated cell sorting to isolate primary human monocytes from samples taken from healthy volunteers and then confirmed that the resulting cell population comprised more than $80 \% \mathrm{CD}^{+} 4^{+}$ monocytes using flow cytometry (Supplementary Figure 11). In real-time chemotaxis assays, both 6-OAU and DL-175 induced migration of human monocytes across a broad range of concentrations, demonstrating for the first time the role of GPR84 in regulating monocyte recruitment (Figure 6e,f). However, the concentration dependence of monocyte migration for 6-OAU and DL-175 is quite different, despite their similar potency in GPR84-CHO cAMP assays. At $1 \mu \mathrm{M}$ and above, for example, DL-175 is a significantly less effective chemoattractant than 6-OAU (Figure 6e), but at lower concentrations DL-175 induces comparable migration (Supplementary Figure 11d). The two structurally distinct GPR84 agonists with relative signaling bias therefore exhibit distinct functional behavior across multiple innate immune cell types, including primary human myeloid cells.

\section{DISCUSSION}

In this study, we report the discovery and characterization of a structurally novel, potent, and selective GPR84 agonist chemical probe. Unlike previously reported ligands, DL-175 shows significant biased signaling at GPR84 in overexpressing cell-lines and induces markedly different signaling in primary murine macrophages and human U937 macrophages in an impedance assay. Through a direct comparison between our new probe and fatty acid mimetic 6-OAU, we show for the first time that activation of GPR84 with different agonists can produce distinct effects in key macrophage functional assays. GPR84 has previously been described as a pro-inflammatory receptor and a potential therapeutic target for chronic 
U937 macrophages
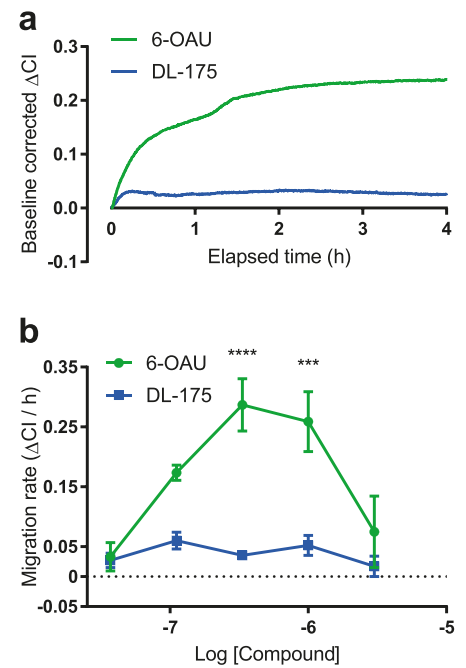

C

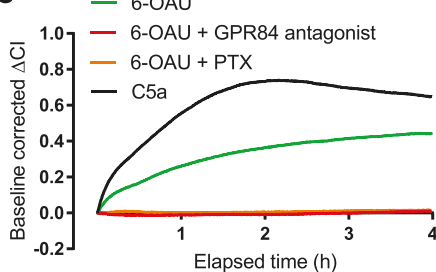

d

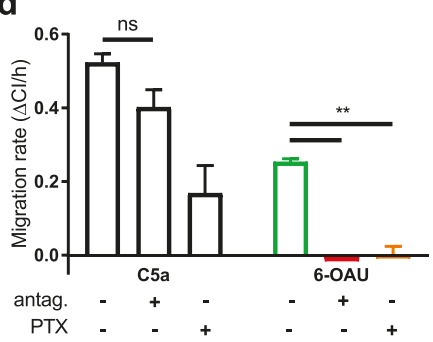

Primary human monocytes

e

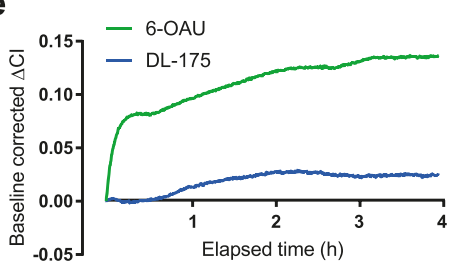

f

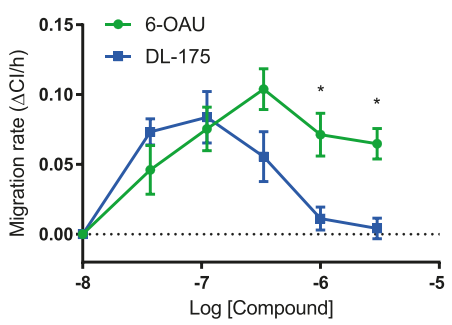

Figure 6. DL-175 is a less effective chemoattractant than 6-OAU for human U937 macrophages and human monocytes. (a) U937 cells migrate toward GPR84 agonists $(1 \mu \mathrm{M})$ in a real time chemotaxis assay, baseline corrected for migration toward vehicle. (b) Dose-response curves for U937 chemotaxis to GPR84 agonists quantified by slope analysis. (c) Real time chemotaxis of U937 cells toward C5a (10 nM) or 6-OAU (333 $\mathrm{nM})$, pretreated with either DMSO for $1 \mathrm{~h}$, GPR84 antagonist $(10 \mu \mathrm{M})$ for $1 \mathrm{~h}$, or PTX $(200 \mathrm{ng} / \mathrm{mL})$ for $2 \mathrm{~h}$. Data shown with each pretreatment group baseline corrected for migration to vehicle. (d) Quantification by slope analysis shows how pretreatments affect migration of U937 cells to C5a $(10 \mathrm{nM})$ or 6-OAU $(333 \mathrm{nM})$. (e) Primary human monocytes migrate toward GPR84 agonists $(1 \mu \mathrm{M})$, baseline corrected for migration toward vehicle. (f) Dose-response curves for primary human monocyte chemotaxis to GPR84 agonists quantified by slope analysis. For panels a, c, and e, data are each representative figures of $n=3$ independent experiments with $2-3$ technical replicates per condition. For panels b, d, and f, data are pooled means \pm SEM from $n=3$ independent experiments and $n=3$ donors for primary monocytes. Statistical analysis was conducted by twoway ANOVA with Sidak's multiple comparisons comparison: $\mathrm{ns}=$ not significant, $* P<0.05, * * P<0.01, * * * P<0.001$, ****P<0.0001 for 6-OAU vs DL-175 in panels $b$ and $f$ and indicated comparisons in panel $d$.

inflammatory diseases through the use of a narrow range of potent synthetic surrogate agonists. ${ }^{9-11,32}$ Our work demonstrates that more diverse small molecule probes can reveal a greater range of GPR84 functional behavior, and we provide a new chemical tool for further elucidation of the receptor's pathophysiological role.

It is important that chemical probes for biological targets are well characterized to avoid drawing erroneous conclusions with low-quality probes that may be unstable, poorly selective, or acting through nonspecific mechanisms. ${ }^{18,33}$ DL-175 is chemically stable across a wide $\mathrm{pH}$ range and therefore suitable for in vitro studies, even if metabolic liabilities may preclude its use in vivo. The off-target effects of GPR84 tool compounds such as embelin $^{34}$ and diindolylmethane ${ }^{19,35}$ (DIM) can confound attempts to study GPR84 biology. Even reported GPR84 probes optimized by medicinal chemistry have shown poor selectivity, as was highlighted recently for DIM derivative PSB16671 , which retained the ability to activate $G_{i}$ proteins in GPR84-KO neutrophils. ${ }^{36}$ We used GPCR screening panels to demonstrate the selectivity of DL-175 and further used genetic knockouts and GPR84 antagonists to demonstrate the GPR84 specificity of the responses elicited by DL-175 in GPR84-CHO cells, U937 cells, and primary murine macrophages. We also report inactive analogue $\mathbf{1 4}$ for use as a structurally matched negative control with DL-175. Further characterization of DL175 as a chemical probe will focus on determining its binding mode to GPR84. As DL-175 was developed through ligand comparison with compounds that occupy the orthosteric binding site, we predict that it also occupies the same pocket with the polar $\mathrm{N}$-oxide motif acting as charge partner for the Arg172 residue involved in MCFA-GPR84 binding. ${ }^{37}$ Future structural studies to confirm the binding mode of DL-175 may provide insight into the origin of signaling bias at GPR84.

We first observed the signaling bias of DL-175 in GPR84 overexpressing cell lines. The enhanced levels of receptor expression and artificial coupling in such systems do not always reflect physiologically relevant cells, exemplified by the observation that GPR84 agonists do not inhibit cAMP production in primary macrophages as seen in recombinant cells but instead potentiate cAMP production through a $\mathrm{G}_{\mathrm{i} \beta \gamma}$ dependent mechanism. ${ }^{11}$ We therefore sought to compare the agonists in more biologically representative systems. Impedance sensing technology allows the dynamic measurement of intracellular signaling using a label-free system appropriate for difficult-to-transfect primary cells. The responses generated by cells in impedance sensing are not always easily attributable to specific pathways, although GPCR mediated impedance signals have been shown to be associated with actin cytoskeletal dynamics through cytochalasin $\mathrm{D}$ inhibition of actin polymerization. ${ }^{22}$ We observed a marked difference in primary murine macrophage and human U937 macrophage responses to the biased ligand that was sensitive to GRK 2/3 inhibition. Notably, in GPR84-CHO cells, the difference in cellular impedance response between GPR84 agonists was not observed (data not shown), suggesting that this difference in response may only manifest in cells with physiological levels of receptor coupling. This further serves to highlight the limitations of comparing agonists with subtle downstream signaling differences in artificial transfected cell systems.

Consistent with their differing impacts on macrophage impedance signaling assays, 6-OAU and G-protein biased DL175 have strikingly different abilities to provoke chemotaxis in human U937 macrophages. This finding is in line with a body 
of evidence implicating $\beta$-arrestin recruitment as a prerequisite for the initiation of chemotaxis. The $\beta$-arrestin dependence of chemotaxis was first shown by the impaired chemotaxis of $\beta$ arrestin 2 deficient lymphocytes toward $\mathrm{CXCL} 12^{38}$ and has subsequently also been demonstrated in non-chemokine GPCRs such as protease-activated receptor 2 (PAR-2) ${ }^{39}$ and angiotensin-II type $1 \mathrm{~A}$ receptor (AT1AR). ${ }^{40}$ Furthermore, the ability of a G-protein biased ligand to induce less chemotaxis by avoiding $\beta$-arrestin recruitment was recently demonstrated by comparing biased ligands at the CXCR3 receptor in human T-cells. ${ }^{41}$ The role of $\beta$-arrestin in macrophage chemotaxis is relatively unexplored, although it has been shown that CCL5 induced chemotaxis of primary human macrophages requires cooperative signaling between G-protein and $\beta$-arrestin pathways. $^{42}$ Interestingly, G-protein biased DL-175 showed a greater capacity to induce chemotaxis in monocytes than macrophages, suggesting that the initiation of monocyte migration is less dependent on $\beta$-arrestin signaling. Functionally selective ligands represent an opportunity to investigate how activation of chemotactic receptors without inducing chemotaxis may affect immune cell behavior. DL-175, for example, could be envisaged to enhance macrophage phagocytosis and clearance of sites of inflammation by efferocytosis without also causing further recruitment of immune cells by chemotaxis.

Over a decade since medium-chain fatty acids (MCFAs) were first established as low-potency agonists for GPR84, it remains unproven as to whether circulating levels of MCFAs can be routinely high enough to activate the receptor in vivo. Dietary MCFAs are believed to be rapidly metabolized in the liver, such that the human plasma concentration of capric acid is generally below $0.5 \mu \mathrm{M}$, a level significantly below that required for appreciable GPR84 activation. ${ }^{43}$ Alternative endogenous GPR84 ligands may activate different signaling pathways or bind allosterically at one of the multiple GPR84 small molecule binding sites. ${ }^{37}$ Precedent for this can be found in the chemokine system, where numerous endogenous ligands behave as biased agonists at the same receptor, creating distinct rather than redundant responses. ${ }^{44,45}$ Moreover, both natural and synthetic chemokine receptor ligands with signaling bias have been demonstrated to induce different cell migratory responses, ${ }^{41,46,47}$ with clear parallels to our study with synthetic GPR84 ligands.

The prospect of alternative, or additional, endogenous GPR84 ligands with signaling bias has far-reaching implications for the therapeutic targeting of the receptor. GPR84 is generally considered a pro-inflammatory receptor and the development of small molecule antagonists for the treatment of chronic inflammatory diseases is ongoing. However, the only clinical test of GPR84 antagonists to-date resulted in failure due to a lack of efficacy in phase II trials for ulcerative colitis, suggesting that the pathophysiological role of GPR84 remains poorly understood. ${ }^{48}$ Other studies instead suggest that GPR84 activation may be beneficial in diseases such as atherosclerosis and Alzheimer's disease. ${ }^{11,49}$ Exploiting functional selectivity at GPR84 may allow agonists to specifically activate beneficial pathways mediated by GPR84, for example, phagocytosis, while avoiding adverse effects. High quality, biased chemical probes for GPR84, such as DL-175, will be invaluable in identifying and validating these alternative therapeutic opportunities.

\section{ASSOCIATED CONTENT}

\section{Supporting Information}

The Supporting Information is available free of charge on the ACS Publications website at DOI: 10.1021/acschembio.9b00533.

Virtual and biological screening data, cAMP data for hit compound, cAMP counterscreening, GPCR selectivity screening, stability of DL-175 and 14, aggregation artifacts in arrestin assay, GPR84 agonist arrestin recruitment, DL-175 G-protein biased signaling, GRK transfection and arrestin recruitment, GRK inhibition and macrophage impedance, U937 cellular impedance, Z-stack image of bead phagocytosis, human monocyte chemotaxis, biological and statistical methods, chemical synthesis, compound characterization, and spectra (PDF)

\section{AUTHOR INFORMATION}

\section{Corresponding Authors}

*E-mail: Angela.Russell@chem.ox.ac.uk.

*E-mail: David.Greaves@path.ox.ac.uk.

ORCID

Maria Chatzopoulou: 0000-0003-1886-7705

Angela J. Russell: 0000-0003-3610-9369

\section{Author Contributions}

D.L. designed the research, synthesized compounds, performed the experiments, analyzed the data, and wrote the paper. L.Z., G.P., and C.R. prepared primary cells and performed flow cytometry experiments. C.J.R.B. synthesized compounds and performed waterLOGSY experiments. M.C. and G.W. supervised the chemistry. A.J.R. and D.R.G. conceived and supervised the project, designed the research, and wrote the paper. All authors edited the manuscript.

\section{Notes}

The authors declare no competing financial interest.

\section{ACKNOWLEDGMENTS}

This work was supported by the BHF Centre of Research Excellence (RE/13/1/30181) and grants from the Novo Nordisk Foundation (NNF15CC0018346) and the British Heart Foundation (RG/15/10/23915).

\section{REFERENCES}

(1) Hauser, A. S., Attwood, M. M., Rask-Andersen, M., Schiöth, H. B., and Gloriam, D. E. (2017) Trends in GPCR drug discovery: new agents, targets and indications. Nat. Rev. Drug Discovery 16, 829-842.

(2) Alvarez-Curto, E., and Milligan, G. (2016) Metabolism meets immunity: The role of free fatty acid receptors in the immune system. Biochem. Pharmacol. 114, 3-13.

(3) Recio, C., Lucy, D., Iveson, P., Iqbal, A. J., Valaris, S., Wynne, G., Russell, A. J., Choudhury, R. P., O'Callaghan, C., Monaco, C., and Greaves, D. R. (2018) The Role of Metabolite-Sensing G ProteinCoupled Receptors in Inflammation and Metabolic Disease. Antioxid. Redox Signaling 29, 237-256.

(4) Burant, C. F., Viswanathan, P., Marcinak, J., Cao, C., Vakilynejad, M., Xie, B., and Leifke, E. (2012) TAK-875 versus placebo or glimepiride in type 2 diabetes mellitus: a phase 2, randomised, double-blind, placebo-controlled trial. Lancet 379, 14031411.

(5) Yousefi, S., Cooper, P. R., Potter, S. L., Mueck, B., and Jarai, G. (2001) Cloning and expression analysis of a novel G protein coupled receptor selectively expressed on granulocytes. J. Leukoc. Biol. 69, $1045-1052$. 
(6) Bouchard, C., Pagé, J., Bédard, A., Tremblay, P., and Vallières, L. (2007) G protein-coupled receptor 84 , a microglia-associated protein expressed in neuroinflammatory conditions. Glia 55, 790-800.

(7) Wang, J., Wu, X., Simonavicius, N., Tian, H., and Ling, L. (2006) Medium-chain fatty acids as ligands for orphan $\mathrm{G}$ protein-coupled receptor GPR84. J. Biol. Chem. 281, 34457-34464.

(8) Lattin, J. E., Schroder, K., Su, A. I., Walker, J. R., Zhang, J., Wiltshire, T., Saijo, K., Glass, C. K., Hume, D. A., Kellie, S., and Sweet, M. J. (2008) Expression analysis of G Protein-Coupled Receptors in mouse macrophages. Immunome Res. 4, 5.

(9) Recio, C., Lucy, D., Purvis, G. S. D., Iveson, P., Zeboudj, L., Iqbal, A. J., Lin, D., O'Callaghan, C., Davison, L., Griesbach, E., Russell, A. J., Wynne, G. M., Dib, L., Monaco, C., and Greaves, D. R. (2018) Activation of the Immune-Metabolic Receptor GPR84 Enhances Inflammation and Phagocytosis in Macrophages. Front. Immunol. 9, 1419.

(10) Suzuki, M., Takaishi, S., Nagasaki, M., Onozawa, Y., Iino, I., Maeda, H., Komai, T., and Oda, T. (2013) Medium-chain Fatty Acidsensing Receptor, GPR84, Is a Proinflammatory Receptor. J. Biol. Chem. 288, 10684-10691.

(11) Gaidarov, I., Anthony, T., Gatlin, J., Chen, X., Mills, D., Solomon, M., Han, S., Semple, G., and Unett, D. J. (2018) Embelin and its derivatives unravel the signaling, proinflammatory and antiatherogenic properties of GPR84 receptor. Pharmacol. Res. 131, 185-198.

(12) Zhang, Q., Yang, H., Li, J., and Xie, X. (2016) Discovery and Characterization of a Novel Small-Molecule Agonist for MediumChain Free Fatty Acid Receptor G Protein-Coupled Receptor 84. J. Pharmacol. Exp. Ther. 357, 337-344.

(13) Liu, Y., Zhang, Q., Chen, L.-H., Yang, H., Lu, W., Xie, X., and Nan, F.-J. (2016) Design and Synthesis of 2-Alkylpyrimidine-4,6-diol and 6-Alkylpyridine-2,4-diol as Potent GPR84 Agonists. ACS Med. Chem. Lett. 7, 579-583.

(14) Pillaiyar, T., Köse, M., Sylvester, K., Weighardt, H., Thimm, D., Borges, G., Förster, I., von Kügelgen, I., and Müller, C. E. (2017) Diindolylmethane Derivatives: Potent Agonists of the Immunostimulatory Orphan G Protein-Coupled Receptor GPR84. J. Med. Chem. 60, 3636-3655.

(15) Labéguère, F., Alvey, L., Newsome, G., Laurent, S., and Fletcher, S. (2013) Novel dihydropyrimidinoisoquinolinones and pharmaceutical compositions thereof for the treatment of inflammatory disorders (gpr84 antagonists). World Patent WO2014095798A1.

(16) Pillaiyar, T., Köse, M., Namasivayam, V., Sylvester, K., Borges, G., Thimm, D., von Kügelgen, I., and Müller, C. E. (2018) 6(Ar)Alkylamino-Substituted Uracil Derivatives: Lipid Mimetics with Potent Activity at the Orphan G Protein-Coupled Receptor 84 (GPR84). ACS Omega 3, 3365-3383.

(17) Violin, J. D., Crombie, A. L., Soergel, D. G., and Lark, M. W. (2014) Biased ligands at G-protein-coupled receptors: promise and progress. Trends Pharmacol. Sci. 35, 308-316.

(18) Blagg, J., and Workman, P. (2017) Choose and Use Your Chemical Probe Wisely to Explore Cancer Biology. Cancer Cell 32, 925.

(19) Yin, H., Chu, A., Li, W., Wang, B., Shelton, F., Otero, F., Nguyen, D. G., Caldwell, J. S., and Chen, Y. A. (2009) Lipid G protein-coupled receptor ligand identification using beta-arrestin PathHunter assay. J. Biol. Chem. 284, 12328-12338.

(20) Owen, S. C., Doak, A. K., Wassam, P., Shoichet, M. S., and Shoichet, B. K. (2012) Colloidal Aggregation Affects the Efficacy of Anticancer Drugs in Cell Culture. ACS Chem. Biol. 7, 1429-1435.

(21) Yu, N., Atienza, J. M., Bernard, J., Blanc, S., Zhu, J., Wang, X., $\mathrm{Xu}, \mathrm{X}$., and Abassi, Y. A. (2006) Real-Time Monitoring of Morphological Changes in Living Cells by Electronic Cell Sensor Arrays: An Approach To Study G Protein-Coupled Receptors. Anal. Chem. 78, 35-43.

(22) Peters, M. F., and Scott, C. W. (2009) Evaluating Cellular Impedance Assays for Detection of GPCR Pleiotropic Signaling and Functional Selectivity. J. Biomol. Screening 14, 246-255.
(23) Grundmann, M., Merten, N., Malfacini, D., Inoue, A., Preis, P., Simon, K., Rüttiger, N., Ziegler, N., Benkel, T., Schmitt, N. K., Ishida, S., Müller, I., Reher, R., Kawakami, K., Inoue, A., Rick, U., Kühl, T., Imhof, D., Aoki, J., König, G. M., Hoffmann, C., Gomeza, J., Wess, J., and Kostenis, E. (2018) Lack of beta-arrestin signaling in the absence of active G proteins. Nat. Commun. 9, 341.

(24) Ikeda, S., Kaneko, M., and Fujiwara, S. (2007) Cardiotonic agent comprising GRK inhibitor. World Patent WO2007034846.

(25) Loniewski, K., Shi, Y., Pestka, J., and Parameswaran, N. (2008) Toll-like receptors differentially regulate GPCR kinases and arrestins in primary macrophages. Mol. Immunol. 45, 2312-2322.

(26) Groot-Kormelink, P. J., Fawcett, L., Wright, P. D., Gosling, M., and Kent, T. C. (2012) Quantitative GPCR and ion channel transcriptomics in primary alveolar macrophages and macrophage surrogates. BMC Immunol. 13, 57.

(27) May, R. C., and Machesky, L. M. (2001) Phagocytosis and the actin cytoskeleton. J. Cell Sci. 114, 1061-1077.

(28) Jones, G. E. (2000) Cellular signaling in macrophage migration and chemotaxis. J. Leukoc. Biol. 68, 593-602.

(29) Iqbal, A. J., Regan-Komito, D., Christou, I., White, G. E., McNeill, E., Kenyon, A., Taylor, L., Kapellos, T. S., Fisher, E. A., Channon, K. M., and Greaves, D. R. (2013) A Real Time Chemotaxis Assay Unveils Unique Migratory Profiles amongst Different Primary Murine Macrophages. PLoS One 8, No. e58744.

(30) Foxman, E. F., Campbell, J. J., and Butcher, E. C. (1997) Multistep navigation and the combinatorial control of leukocyte chemotaxis. J. Cell Biol. 139, 1349-1360.

(31) Ross, R. (1999) Atherosclerosis-An Inflammatory Disease. N. Engl. J. Med. 340, 115-126.

(32) Wei, L., Tokizane, K., Konishi, H., Yu, H.-R., and Kiyama, H. (2017) Agonists for G-protein-coupled receptor 84 (GPR84) alter cellular morphology and motility but do not induce pro-inflammatory responses in microglia. J. Neuroinflammation 14, 198.

(33) Arrowsmith, C. H., Audia, J. E., Austin, C., Baell, J., Bennett, J., Blagg, J., Bountra, C., Brennan, P. E., Brown, P. J., Bunnage, M. E., Buser-Doepner, C., Campbell, R. M., Carter, A. J., Cohen, P., Copeland, R. A., Cravatt, B., Dahlin, J. L., Dhanak, D., Edwards, A. M., Frederiksen, M., Frye, S. V., Gray, N., Grimshaw, C. E., Hepworth, D., Howe, T., Huber, K. V. M., Jin, J., Knapp, S., Kotz, J. D., Kruger, R. G., Lowe, D., Mader, M. M., Marsden, B., Mueller-Fahrnow, A., Müller, S., O’Hagan, R. C., Overington, J. P., Owen, D. R., Rosenberg, S. H., Ross, R., Roth, B., Schapira, M., Schreiber, S. L., Shoichet, B., Sundström, M., Superti-Furga, G., Taunton, J., Toledo-Sherman, L., Walpole, C., Walters, M. A., Willson, T. M., Workman, P., Young, R. N., and Zuercher, W. J. (2015) The promise and peril of chemical probes. Nat. Chem. Biol. 11, 536-541.

(34) Nikolovska-Coleska, Z., Xu, L., Hu, Z., Tomita, Y., Li, P., Roller, P. P., Wang, R., Fang, X., Guo, R., Zhang, M., Lippman, M. E., Yang, D., and Wang, S. (2004) Discovery of embelin as a cellpermeable, small-molecular weight inhibitor of XIAP through structure-based computational screening of a traditional herbal medicine three-dimensional structure database. J. Med. Chem. 47, $2430-2440$

(35) Chen, I., Safe, S., and Bjeldanes, L. (1996) Indole-3-carbinol and diindolylmethane as aryl hydrocarbon $(\mathrm{Ah})$ receptor agonists and antagonists in T47D human breast cancer cells. Biochem. Pharmacol. 51, 1069-1076.

(36) Mancini, S. J., Mahmud, Z. Al, Jenkins, L., Bolognini, D., Newman, R., Barnes, M., Edye, M. E., McMahon, S. B., Tobin, A. B., and Milligan, G. (2019) On-target and off-target effects of novel orthosteric and allosteric activators of GPR84. Sci. Rep. 9, 1861.

(37) Mahmud, Z. Al, Jenkins, L., Ulven, T., Labéguère, F., Gosmini, R., De Vos, S., Hudson, B. D., Tikhonova, I. G., and Milligan, G. (2017) Three classes of ligands each bind to distinct sites on the orphan G protein-coupled receptor GPR84. Sci. Rep. 7, 17953.

(38) Fong, A. M., Premont, R. T., Richardson, R. M., Yu, Y.-R. A., Lefkowitz, R. J., and Patel, D. D. (2002) Defective lymphocyte chemotaxis in beta-arrestin2- and GRK6-deficient mice. Proc. Natl. Acad. Sci. U. S. A. 99, 7478-7483. 
(39) Zoudilova, M., Min, J., Richards, H. L., Carter, D., Huang, T., and DeFea, K. A. (2010) beta-Arrestins scaffold cofilin with chronophin to direct localized actin filament severing and membrane protrusions downstream of protease-activated receptor-2. J. Biol. Chem. 285, 14318-14329.

(40) Hunton, D. L., Barnes, W. G., Kim, J., Ren, X.-R., Violin, J. D., Reiter, E., Milligan, G., Patel, D. D., and Lefkowitz, R. J. (2005) Betaarrestin 2-dependent angiotensin II type $1 \mathrm{~A}$ receptor-mediated pathway of chemotaxis. Mol. Pharmacol. 67, 1229-1236.

(41) Smith, J. S., Nicholson, L. T., Suwanpradid, J., Glenn, R. A., Knape, N. M., Alagesan, P., Gundry, J. N., Wehrman, T. S., Atwater, A. R., Gunn, M. D., MacLeod, A. S., and Rajagopal, S. (2018) Biased agonists of the chemokine receptor CXCR3 differentially control chemotaxis and inflammation. Sci. Signaling 11, No. eaaq1075.

(42) Cheung, R., Malik, M., Ravyn, V., Tomkowicz, B., Ptasznik, A., and Collman, R. G. (2009) An arrestin-dependent multi-kinase signaling complex mediates MIP-1 $\beta /$ CCL4 signaling and chemotaxis of primary human macrophages. J. Leukocyte Biol. 86, 833-845.

(43) Shrestha, R., Hui, S.-P., Imai, H., Hashimoto, S., Uemura, N., Takeda, S., Fuda, H., Suzuki, A., Yamaguchi, S., Hirano, K., and Chiba, H. (2015) Plasma capric acid concentrations in healthy subjects determined by high-performance liquid chromatography. Ann. Clin. Biochem. 52, 588-596.

(44) Leach, K., Charlton, S. J., and Strange, P. G. (2007) Analysis of second messenger pathways stimulated by different chemokines acting at the chemokine receptor CCR5. Biochem. Pharmacol. 74, 881-890.

(45) Zweemer, A. J. M., Toraskar, J., Heitman, L. H., and IJzerman, A. P. (2014) Bias in chemokine receptor signalling. Trends Immunol. $35,243-252$.

(46) Corbisier, J., Huszagh, A., Galés, C., Parmentier, M., and Springael, J.-Y. (2017) Partial Agonist and Biased Signaling Properties of the Synthetic Enantiomers J113863/UCB35625 at Chemokine Receptors CCR2 and CCR5. J. Biol. Chem. 292, 575-584.

(47) Rajagopal, S., Bassoni, D. L., Campbell, J. J., Gerard, N. P., Gerard, C., and Wehrman, T. S. (2013) Biased agonism as a mechanism for differential signaling by chemokine receptors. J. Biol. Chem. 288, 35039-35048.

(48) Vermeire, S., Reinisch, W., Wasko-Czopnik, D., Van Kaem, T., Desrivot, J., Vanhoutte, F., and Beetens, J. (2017) P610 Efficacy and safety of GLPG1205, a GPR84 antagonist, in ulcerative colitis: multicentre proof-of-concept study. J. Crohn's Colitis 11, S390-S391.

(49) Audoy-Rémus, J., Bozoyan, L., Dumas, A., Filali, M., Lecours, C., Lacroix, S., Rivest, S., Tremblay, M.-E., and Vallières, L. (2015) GPR84 deficiency reduces microgliosis, but accelerates dendritic degeneration and cognitive decline in a mouse model of Alzheimer's disease. Brain, Behav., Immun. 46, 112-120. 